
IS PROTECTIVE AGAINST CALCIFIC TENDINOPATHY (CT)

I. Bernabei ${ }^{1}$, D. Kronenberg ${ }^{2}$, R. Stange ${ }^{2}$, J. Bertrand ${ }^{3}$, T. Hügle ${ }^{1}$, A. So $^{1}$, N. Busso ${ }^{1}, \mathrm{~S}$. Nasi ${ }^{1}{ }^{1}$ Centre Hospitalier Universitaire Vaudois and University of Lausanne, Department of Musculoskeletal Medicine, Service of Rheumatology, Lausanne, Switzerland; ${ }^{2}$ Institute of Musculoskeletal Medicine, Westfaelische Wilhelms University Muenster, Department of Regenerative Musculoskeletal Medicine, Münster, Germany; ${ }^{3}$ Otto-von-Guericke University, Department of Orthopaedic Surgery, Magdeburg, Germany

Background: Pathological (or heterotopic) calcification is the deposition of calcium-containing crystals in soft tissues that normally do not calcify. The deposition of these crystals in tendons such as the rotator cuff and the Achilles tendon is known as calcific tendinopathy (CT). CT is a painful condition, which increases tendon rupture rate and leads to disability.

Objectives: To understand what inhibits calcification, in order to provide new strategies to treat a condition for which existing therapies are ineffective.

Methods: We investigated the role of the gasotransmitter hydrogen sulfide $\left(\mathrm{H}_{\mathrm{S}} \mathrm{S}\right)$, and in particular of the $\mathrm{H}_{2} \mathrm{~S}$-producing enzyme cystathionine $\gamma$-lyase (CSE) in CT. In vitro, we induced calcification in tenocytes from WT and CSE KO mice or we treated WT tenocytes with different $\mathrm{H}_{2} \mathrm{~S}$ donors. In vivo, calcification was assessed in a surgery-induced murine model of CT (tenotomy of the Achilles tendon) and in a spontaneous model of CT (aging). Samples obtained from patients with rotator cuff or Achilles tendon CT were also analyzed. To investigate the underlying mechanisms of the CSE- $\mathrm{H}_{2} \mathrm{~S}$ effect, we focused on the bone morphogenic proteins (BMPs) pathway. We additionally explored if altered extracellular matrix (ECM) organization, due to lysyl oxidase (LOX) activity and aberrant collagen-crosslinks, could also be involved in CT. In this context, we studied if $\mathrm{H}_{2} \mathrm{~S}$ could affect LOX expression and activity.

Results: In vitro, tenocyte calcification was inhibited by exogenous $\mathrm{H}_{2} \mathrm{~S}$-donors, while it was exacerbated in CSE KO tenocytes. The protective role of CSE- $\mathrm{H}_{2} \mathrm{~S}$ was confirmed in vivo. In aged mice, microtomography analysis revealed exacerbated Achilles tendon calcification in CSE KO mice compared to WT. In the surgery-induced model of CT, an inverse correlation between calcification and CSE expression in operated Achilles tendon was seen over time. Similarly, inversed correlation between calcification and CSE expression was found in human CT samples.

Reduced calcification in tenocytes exposed to $\mathrm{H}_{2} \mathrm{~S}$ was accompanied by decreased expression of genes coding for BMP2, BMP4 and decreased activation of the BMP signaling pathway (pSMAD1/5/8). On the contrary, BMPs expression and BMPs-pathway activation were exacerbated in CSE KO tenocytes compared to WT tenocytes.

We next investigated whether ECM disorganization could play a role in CT. Tenocytes cultured in calcification media and treated with the pan-inhibitor of lysyl oxidases (LOX, LOXL1-4) $\beta$-aminopropionitrile (BAPN) showed decreased calcification. This pointed to a potential beneficial role of LOX inhibition, therefore decreased collagen-crosslinks, in CT. By analysis of LOXs gene expression in WT and CSE KO tenocytes cultured in calcifying condition, we found much higher expression (4-fold) of LOX, LOL2 and LOXL4 in CSE KO tenocytes. Moreover, $\mathrm{H}_{2} \mathrm{~S}$-donors inhibited LOX activity. Altogether, these results suggest that decreased $\mathrm{H}_{2} \mathrm{~S}$ could lead to aberrant LOX expression and activity, excessive collagen cross-links in the ECM, and ultimately calcification. Further experiments are ongoing to prove these hypotheses.

Conclusion: We suggest targeting $\mathrm{H}_{2} \mathrm{~S}$ production by CSE, or supplying an $\mathrm{H}_{2} \mathrm{~S}$-donor, is of therapeutic relevance to pathological calcification in the context of $\mathrm{CT}$ and can modify its disease course.

The anti-mineralizing effect of $\mathrm{H}_{2} \mathrm{~S}$ in tendons could be due to both inhibition of the BMPs pathway and suppression of abnormal LOXs activity.

Disclosure of Interests: None declared.

DOI: 10.1136/annrheumdis-2021-eular.2419

\section{POS0042 NOTCH 1 INHIBITION INCREASES OSTEOCLAST PROGENITOR ACTIVITY IN THE MOUSE MODEL OF RHEUMATOID ARTHRITIS}

M. Filipović ${ }^{1}$, A. Šućur ${ }^{1}$, D. Flegar ${ }^{1}$, Z. Jajić ${ }^{2}$, M. Ikić Matijašević ${ }^{3}$, N. Lukač ${ }^{1}$, N. Kovačici ${ }^{1}$, T. Kelava ${ }^{1}$, D. Šisl ${ }^{1}$, K. Zrinski Petrović ${ }^{1}$, V. Katavić ${ }^{1}$, D. Grčević ${ }^{1}$ ${ }^{1}$ University of Zagreb School of Medicine, Croatian Institute for Brain Research, Laboratory for Molecular Immunology, Zagreb, Croatia; ${ }^{2}$ University of Zagreb School of Medicine, Clinical Hospital Center "Sestre Milosrdnice,' Department of Rheumatology, Physical Medicine and Rehabilitation, Zagreb, Croatia; ${ }^{3}$ University of Zagreb School of Medicine, Clinical Hospital "Sveti Duh", Department of Clinical Immunology and Allergology, Zagreb, Croatia

Background: Osteoclasts mediate periarticular and systemic bone loss in rheumatoid arthritis (RA). Osteoclast progenitor cells (OCPs) derived from the myeloid lineage are susceptible to regulation through Notch signaling. Murine

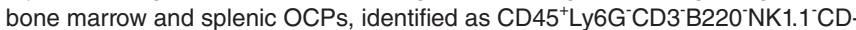
$11 \mathrm{~b}^{\mathrm{lo} /+} \mathrm{CD} 115^{+} \mathrm{CCR} 2^{+}$cells, are specifically increased in arthritis. We previously identified an increased frequency of OCPs expressing Notch receptors in arthritic mice.

Objectives: Several studies suggested that Notch signaling modulation affects the course of experimental arthritis. We aimed to determine the effects of Notch receptor signaling inhibition on OCP activity and arthritis severity in murine collagen-induced arthritis $(\mathrm{CIA})$.

Methods: Male C57/BI6 and DBA mice were immunized with chicken type II collagen and treated with i.p. injections of anti-Notch 1 neutralizing antibodies $(1 \mathrm{mg} / \mathrm{kg})$. Notch receptor 1 through 4 expression on OCPs was analyzed by flow cytometry in periarticular bone marrow (PBM) and spleen (SPL). Gene expression of Notch receptors, ligands and transcription targets as well as osteoclas differentiation genes RANK, cFos and cFms was determined by qPCR from tissues and sorted OCPs. FACS sorted OCPs were stimulated by osteoclastogenic factors (M-CSF and RANKL), in control, IgG, Jagged (Jag) 1 or Delta-like (DLL)1 coated wells, with or without anti-Notch 1 antibodies. Research was approved by the Ethics Committee.

Results: We confirmed the expression of Notch receptors on OCPs by flow cytometry with Notch 1 and 2 being most abundantly expressed (around 25\% and $40 \%$ positive OCPs in PBM and $35 \%$ and $20 \%$ in SPL respectively), with a significant increase of Notch 2 expression in arthritis. Seeding OCPs on DLL1 coated wells significantly increased while seeding on Jag1 coated wells significantly decreased osteoclastogenesis as reflected on the number of TRAP+ osteoclasts and expression of osteoclast differentiation genes. The addition of anti-Notch 1 antibodies to ligand-stimulated OCPs resulted in an increased number of TRAP+ osteoclasts, partially reversing Jag1 inhibition. In vivo treatment with anti-Notch 1 antibodies did not affect total OCP frequency, but increased expression of Notch 4 both in PBM and SPL as seen by flow cytometry and qPCR. Additionally, anti-Notch 1 treatment stimulated Notch transcription factors HES and HEY. Both PBM and SPL cultured OCPs from anti-Notch 1 treated mice produced a higher number of large TRAP+ osteoclasts, doubling the area covered with osteoclasts in the latter compared to untreated mice. Increased osteoclastogenesis in vitro was further confirmed by an increased expression of osteoclast differentiation genes in the treated group.

Conclusion: Our results confirm that Notch signaling may represent an important therapeutic target for the regulation of osteoclast activity in arthritis. Both in vitro and in vivo anti-Notch 1 neutralizing antibodies enhanced osteoclastogenesis in CIA model, implying an inhibitory role of Notch 1 signaling in osteoclast differentiation. As Notch 2 expression is increased on OCPs of arthritic mice, we next plan to determine the effects of Notch 2 neutralization on osteoclast activity and arthritis severity.

\section{REFERENCES:}

[1] Ikić Matijašević M, Flegar D, Kovačić N, Katavić V, Kelava T, Šućur A, et al. Increased chemotaxis and activity of circulatory myeloid progenitor cells may contribute to enhanced osteoclastogenesis and bone loss in the C57BL/6 mouse model of collagen-induced arthritis. Clin Exp Immunol. 2016;186(3):321-35.

[2] Šućur A, Filipović M, Flegar D, Kelava T, Šisl D, Lukač N, et al. Notch receptors and ligands in inflammatory arthritis - a systematic review. Immunology Letters 2020 Vol. 223, p. 106-14.

Acknowledgements: The work has been supported by Croatian Science Foundation projects IP-2018-01-2414, UIP-2017-05-1965 and DOK-2018-09-4276.

Disclosure of Interests: None declared.

DOI: 10.1136/annrheumdis-2021-eular.2601

\begin{tabular}{|l|l}
\hline POS0043 & PERIPHERAL QUANTITATIVE COMPUTED \\
& TOMOGRAPHY IN THE ASSESSMENT OF BONE \\
& MINERAL DENSITY IN ANTI-TNF-TREATED \\
& RHEUMATOID ARTHRITIS AND ANKYLOSING \\
& SPONDYLITIS PATIENTS
\end{tabular}

B. Juhász ${ }^{1}$, K. Gulyás², Á. Horváth² ${ }^{2}$ E. Végh ${ }^{2}$, A. Pusztai² ${ }^{2}$ A. Szentpetery ${ }^{3}$, Z. Pethö ${ }^{2}$, N. Bodnár ${ }^{2}$, A. Hamar ${ }^{2}$, L. Bodoki ${ }^{2}$, H. P. Bhattoa ${ }^{4}$, É . Szekanecz ${ }^{1}$, K. Hodosi ${ }^{2}$, A. Domjan², S. Szamosi², C. Horváth ${ }^{5}$, S. Szántó ${ }^{2}$, G. Szücs², H. Raterman ${ }^{6}$, W. Lems ${ }^{7}$, O. Fitzgerald ${ }^{8}, Z$ Z. Szekanecz ${ }^{2}{ }^{1}$ University of Debrecen, Oncology, Debrecen, Hungary; ${ }^{2}$ University of Debrecen, Rheumatology, Debrecen, Hungary; ${ }^{3}$ Uppsala University Hospital, Rheumatology, Uppsala, Sweden; ${ }^{4}$ University of Debrecen, Laboratory Medicine, Debrecen, Hungary; ${ }^{5}$ Semmelweis University, Medicine, Budapest, Hungary; ${ }^{6}$ Northwest Clinics, Rheumatology, Alkmaar, Netherlands; ${ }^{7}$ University of Amsterdam, Rheumatology, Amsterdam, Netherlands; ${ }^{8}$ University College Dublin, Conway Institute for Biomolecular Research, Dublin, Ireland

Background: Rheumatoid arthritis (RA) and ankylosing spondylitis (AS) have been associated with osteoporosis. There have been very few data on the use of peripheral quantitative computed tomography (QCT) in anti-TNF-treated patients. 
Objectives: We wished to assess volumetric bone mineral density (BMD) by forearm QCT in conjunction with dual-energy X-ray absorptiometry (DXA) and bone biomarkers in RA and AS.

Methods: Forty RA and AS patients treated with etanercept (ETN) or certolizumab pegol (CZP) were included in a 12-month follow-up study. Peripheral QCT and DXA BMD were determined. Bone biomarkers, such as PTH, osteocalcin, RANKL, 25-hydroxyvitamin D (VITD), P1NP, CTX, sclerostin, DKK-1 and cathepsin K (CATHK) were assessed by ELISA.

Results: There was no further bone loss during anti-TNF treatment. Volumetric and areal BMD showed significant correlations with each other $(p<0.05)$. Total QCT BMD after 12 months was inversely determined by disease activity at baseline in the full cohort $(p=0.030)$. Cortical BMD was negatively determined by baseline disease activity $(p=0.005)$ and CATHK $(p=0.025)$. In RA, VITD-0 determined QTRABBMD-12 ( $p=0.005)$. In the full cohort, the one-year change in QTRABBMD was related to TNF inhibition together with higher VITD-0 $(p=0.031)$. Therapy and lower CATHK determined QCORTBMD changes $(p=0.006)$. In RA, treatment together with VITD-0 $(p<0.01)$ or CATHK-0 $(p=0.002)$, while in AS, treatment together with RANKL-0 $(p<0.05)$ determined QCT BMD changes. Conclusion: QCT confirmed that biologics may attenuate bone loss. Disease activity, CATHK, RANKL and VITD may predict the effects of anti-TNF treatment on volumetric BMD changes. There may be differences between RA and AS in this respect.

Acknowledgements: This research was supported by Hungarian National Scientific Research Fund (OTKA) grant No. K 105073 (H.P.B. and Z.S.); by the European Union and the State of Hungary and co-financed by the European Social Fund in the framework of TAMOP-4.2.4.A/2-11/1-2012-0001 'National Excellence Program '(Z.S.); by the European Union grant GINOP-2.3.2-15-2016-00050 (Z.S.); and by the Pfizer Investigator Initiated Research Grants no. WS1695414 and WS1695450 (Z.S.).

Disclosure of Interests: Balázs Juhász: None declared, Katalin Gulyás: None declared, Ágnes Horváth: None declared, Edit Végh: None declared, Anita Pusztai: None declared, Agnes Szentpetery: None declared, Zsófia Pethö: None declared, Nóra Bodnár: None declared, Attila Hamar: None declared, Levente Bodoki: None declared, Harjit Pal Bhattoa: None declared, Éva Szekanecz: None declared, Katalin Hodosi: None declared, Andrea Domjan: None declared, Szilvia Szamosi Speakers bureau: Roche, Csaba Horváth: None declared, Sándor Szántó Speakers bureau: Abbvie, MSD, Novartis, Consultant of: Abbvie, Novartis, Gabriella Szücs Speakers bureau: Roche, Boehringer, Actelion, Sager, Consultant of: Actelion, Boehringer, Hennie Raterman: None declared, WIllem Lems Speakers bureau: Pfizer, Amgen, Lilly, UCB, Galapagos, Consultant of: Pfizer, Amgen, Lilly, UCB, Galapagos, Oliver FitzGerald Speakers bureau: AbbVie, Janssen, Pfizer, Consultant of: BMS, Celgene, Eli Lilly, Janssen, Pfizer, Grant/research support from: AbbVie, BMS, Eli Lilly, Novartis, Pfizer, Zoltán Szekanecz Speakers bureau: Pfizer, Roche, Abbvie, Novartis, Lilly, Sanofi, Consultant of: Pfizer, Abbvie, Novartis, Grant/research support from: Pfizer, UCB.

DOI: 10.1136/annrheumdis-2021-eular.1951

\section{POS0044 T315 SUPPRESSES OSTEOGENIC DIFFERENTIATION IN SAOS-2 CELLS BY INHIBITING PHOSPHORYLATION OF AKT}

Z. Huang ${ }^{1}$, X. Huang ${ }^{1}$, Y. Huang ${ }^{1}$, Z. Li ${ }^{1}$, Q. Huang ${ }^{1}$, T. Li ${ }^{1} .{ }^{1}$ Guangdong Second Provincial General Hospital, Department of Rheumatology and Immunology, Guangzhou, China

Background: New bone formation is common in the late stage of various inflammatory arthritis, while osteoblasts play a vital role in this process. Activation of $\mathrm{PI} 3 \mathrm{~K} /$ Akt pathway promotes the differentiation and enhances the function of osteoblasts [1]. T315 is a novel small molecule drug, which may induce apoptosis and suppress the expression of cellular markers of chronic lymphocytic leukemia cells by disrupting PI3K/ Akt pathway [2]. However, the lack of study focuses on the influence of T31T on the other cells, except tumor cell lines.

Objectives: We aimed to assess the effect of T315 on human osteoblast-like Saos-2 cells, while its potential mechanism in PI3K/ Akt pathway was evaluated as well.

Methods: (1) Saos-2 was stimulated with an osteogenic reagent which contained L-ascorbic acid, $\beta$-glycerophosphoric acid, and dexamethasone. The concentration of T315 was adjusted to $0 \mu \mathrm{g} / \mathrm{ml}, 1 \mu \mathrm{g} / \mathrm{ml}$, and $2 \mu \mathrm{g} / \mathrm{ml}$ in the culture medium. (2) Alizarin red stain and alkaline phosphatase (ALP) stain were performed at d0, d7, d14, and d21 after being treated with T315. (3) Cellular protein was extracted at $\mathrm{d} 0, \mathrm{~d} 3$, and $\mathrm{d} 6$ after being treated with T315, then ALP activity was tested based on a recommendation from the manufacturer of the kit. (4) Collagen type $1 \alpha 2$ Chain (Col1 $\alpha 2)$ and osteocalcin (OCN), two osteogenic markers, were measured through western blot, with glyceraldehyde 3-phosphate dehydrogenase (GAPDH) as an endogenous control. (5) Phospho-phosphoinositide 3-kinase ( $\mathrm{pPI} 3 \mathrm{~K})$, phospho-protein kinase $\mathrm{B}$ (pAkt), and runt-related transcription factor 2 (Runx2) was tested through western blot as well. GAPDH or protein kinase (Akt) was chosen as an internal reference as appropriate. (6) Analysis of variance with the least significant difference was used to analyze the data. A $P<0.05$ was considered statistically significant.

Results: (1) The higher concentration of T315 related to the less relative mineralized area and the positive area of ALP, while longer incubation time with T315 decreased these regions as well (Figure 1A-C). (2) T315 reduced the activity of ALP accordingly (Figure 1D). (3) T315 suppressed the protein expression of Col1 $\alpha 2$ and OCN in a dose-dependent and time-dependent manner (Figure 1E, F). (4) T315 did not alter pPI3K, but it inhibited the phosphorylation of Akt (Fig ure $1 \mathrm{G}, \mathrm{H})$. (5) Runx2 was reduced because of the greater dose or longer incubation time with T315 (Figure 1).

Conclusion: T315 inhibits the differentiation of osteoblasts through inhibiting the phosphorylation of Akt. Surprisingly, pPI3K seldom changes in this process, so its detail mechanism should be investigated in further.

REFERENCES:

[1] Exp Biol Med (Maywood) 2020;245(6):552-561.

[2] Blood 2015;125(2):284-295.
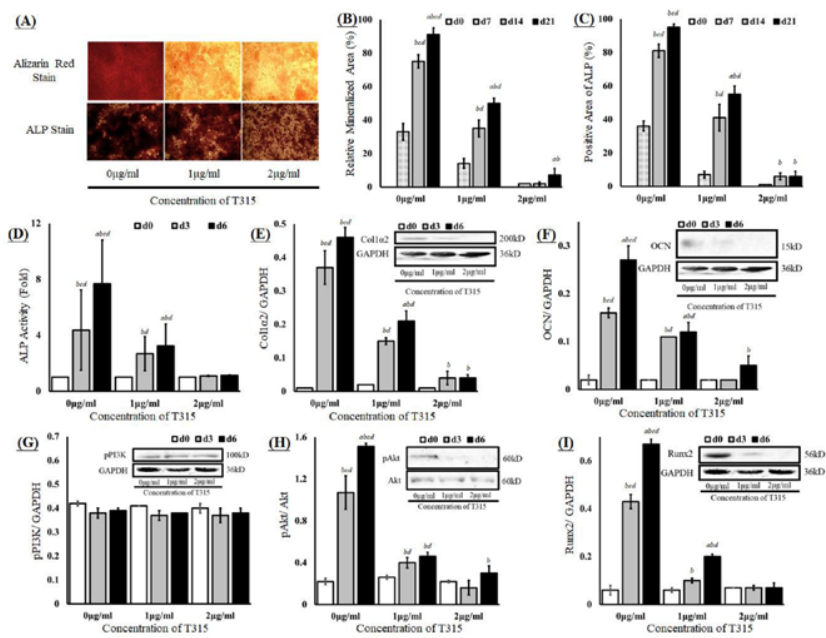

Figure 1. Effect of T315 on Saos-2 cells and PI3K/Akt pathway. (A) Alizarin red stain and ALP stain at d21. (B) Relative mineralized area in Alizarin red stain. (C) Positive area in ALP stain (D) ALP activity. Western blot analysis and its bands at d6 for Col1 $\alpha 2(E), O C N(F), p P I 3 K$ (G), pAkt $(\mathrm{H})$, and Runx2 (I). Results were normalized by GAPDH or Akt.ALP: Alkaline phosphatase; Col1 $\alpha 2$ : Collagen type $1 \alpha 2$ Chain; GAPDH: Glyceraldehyde 3-phosphate dehydrogenase; OCN: Osteocalcin; pPI3K: Phospho-phosphoinositide 3-kinase; pAkt: Phospho-protein kinase B; Akt: Protein kinase; Runx2: Runt-related transcription factor 2. ${ }^{a}$ Compared with d3 in the same concentration of T315, $P<0.05 .^{b}$ Compared with d0 in the same concentration of T315, $P<0.05 .{ }^{\circ}$ Compared with $1 \mu \mathrm{g} / \mathrm{ml} \mathrm{T} 315$ in the same incubation time, $P<0.05 .{ }^{d}$ Compared with $2 \mu \mathrm{g} / \mathrm{ml}$ T315 in the same incubation time, $P<0.05$.

Disclosure of Interests: None declared.

DOI: 10.1136/annrheumdis-2021-eular.2428

\section{POS0045 LEAST SIGNIFICANT CHANGE IN BONE DENSITOMETRY IN PATIENTS WITH OBESITY}

D. Claire ${ }^{1}$, M. Geoffroy ${ }^{1}$, L. Kanagaratnam ${ }^{2}$, C. Isabelle ${ }^{1}$, A. Hittinger ${ }^{1}$, B. Loïs ${ }^{1}$, C. Clément ${ }^{1}$, J. H. Salmon ${ }^{1} .{ }^{1}$ Centre Hospitalier Universitaire de Reims, Rheumatology, Reims, France; ${ }^{2}$ Centre Hospitalier Universitaire de Reims, Unité d'aide méthologique, Reims, France

Background: Dual energy X-ray absoprtiometry is the reference method to mesure bone mineral density (1). Loss of bone mineral density is significant if it exceeds the least significant change. The threshold value used in general population is $0,03 \mathrm{~g} / \mathrm{cm}^{2}(2)$. Patients with obesity are known for having a higher bone mineral density due to metabolism and physiopathology characteristics $(3,4)$. Objectives: The aim of our study was to determine the least significant change in bone densitometry in patients with obesity.

Methods: We conducted an interventionnal study in 120 patients with obesity who performed a bone densitometry. We measured twice the bone mineral density at the lumbar spine, the femoral neck and the total hip in the same time $(5,6)$ We determined the least significant change in bone densitometry from each pair of measurements, using the Bland and Altman method. We also determined the least significant change in bone densitometry according to each stage of obesity. Results: The least significant change in bone densitometry in patients with obesity is $0,046 \mathrm{~g} / \mathrm{cm}^{2}$ at the lumbar spine, $0.069 \mathrm{~g} / \mathrm{cm}^{2}$ at the femoral neck and $0.06 \mathrm{~g} / \mathrm{cm}^{2}$ at the total hip. 